Sharif University of Technology
Scientia Iranica
SCIENTIA
I RAN I CA

\title{
Petahertz-frequency plasmons in graphene nanopore and their application to nanoparticle sensing
}

\author{
B. Fotouhi, V. Ahmadi*, M. Abasifard and V. Faramarzi \\ Department of Electrical \& Computer Engineering, Tarbiat Modares University, Tehran, Iran.
}

Received 17 June 2016; received in revised form 27 February 2017; accepted 20 May 2017

\section{KEYWORDS}

Interband plasmon;

Graphene;

Nanopore;

Nanoparticle;

Discrete Dipole

Approximation

(DDA).

\begin{abstract}
The Surface Plasmon Resonance (SPR) properties in the petahertz $\left(10^{15} \mathrm{~Hz}\right)$ frequency range for monolayer graphene nanosheet and graphene nanopore are investigated using discrete dipole approximation method. We calculate graphene refractive indices by using first-principle density functional theory. The near-field enhancement made by plasmon in these structures is studied by employing finite-difference-time-domain method. For graphene nanosheets, energy of the SPR peak drops with increase in the sheet length. Also, for graphene nanopores smaller than $5 \mathrm{~nm}$ in length, increasing the pore diameter decreases energy of the SPR peak and for some length values like $6 \mathrm{~nm}$, this energy value is raised. For larger sheets (e.g., $8 \mathrm{~nm}$ ), SPR peak is rather unchanged by variations of the pore diameter. The SPR is used to detect nanoscale objects such as gold, silver, copper, rhodium, and aluminium oxide. If nanoscale particles are inserted into the graphene nanopore, 0.195 to $0.474 \mathrm{eV}$ shift in the SPR spectra appears. Type of the presented nanoparticle can be clearly determined by measuring the energy shifts in the SPR spectra. Our results show that petahertz-frequency plasmon in graphene nanopore can be used as a nanoscale-object detection methodology.
\end{abstract}

(C) 2017 Sharif University of Technology. All rights reserved.

\section{Introduction}

Graphene has shown excellent mechanical flexibility, biocompatibility, high electron mobility, and unique plasmon properties [1-7]. Recently, graphene has been utilized as pressure sensor [8], dipolar-antenna in the terahertz frequency range [9], and membrane for DNA translocation detection [10]. Surface Plasmon Resonances (SPR) and plasmon propagation in graphene have attracted attention due to their great application in nano-photonics, biology, sensing, gap detection, and DNA sensing [8,11-13]. Different types of the plasmon in graphene are: (1) collective-intraband excitation of the conductance electrons with $0-3 \mathrm{eV}$ energy, (2) collective-interband excitations of the $\pi$ valance electrons with 4-12 eV energy, and (3) collective-interband

\footnotetext{
*. Corresponding author. Tel./Fax: +982182883368
} E-mail address: v_ahmadi@modares.ac.ir (V. Ahmadi) excitations of all valence electrons $(\pi+\sigma$ plasmon $)$ with 14-33 eV energy [14-21]. Current studies show that interband $\pi$ plasmon in nanometer-size graphene ribbons can be tuned by changing the ribbon size [22]. By the presence of single-point defect, interband $\pi+\sigma$ plasmon can be locally enhanced at an atomic scale to yield unprecedented levels of field confinement (about $0.2 \mathrm{~nm}$ ) [23]. The SPR properties of interband $\pi$ plasmon of monolayer and multilayer graphene nanodiscs show strong small-size and field-enhancement effects [24]. Also, the interband $\pi$ plasmon resonance behaviour for the graphene nanosheets and graphene nanopores, and their application to DNA nucleotide sensing have been investigated $[11,12]$. The SPR properties of the interband $\pi$ plasmons in graphene nanopore can be used in near future as a new methodology for DNA sequencing or other single-molecule detection mechanisms [11,12]. This is because of the fact that interband $\pi$ and $\pi+\sigma$ plasmons in graphene 
are strongly localized and plasmon wavelength is about $\lambda_{0} / 200$, where $\lambda_{0}$ is the free-space wavelength equal to $\sim 250$ and $\sim 80 \mathrm{~nm}$ for the interband $\pi$ and $\pi+\sigma$ plasmons in graphene, respectively. Hence, both types of the interband plasmons in graphene are very strong, sensitive to small and inert to large dimensions and they can be used in nanoscale-object sensing, counting, and manipulation.

In this research, first we calculate refractive indices for graphene by using first-principle Density Functional Theory (DFT) at petahertz frequency range (corresponding to the interband $\pi+\sigma$ plasmon). Then, we analyze interband $\pi+\sigma$ plasmon properties and corresponding Electric Field Enhancement (EFE) of graphene nanosheet using Discrete Dipole Approximation (DDA) and Finite-Difference-Time-Domain (FDTD) methods $[25,26]$. In addition, we look into the effect of making a nanopore and changing its diameter at the center of the graphene nanosheet. Also, we investigate the possibility of the petahertz-frequency plasmons to be used in nanoscale-particle sensing; like gold $(\mathrm{Au})$, silver $(\mathrm{Ag})$, rhodium $(\mathrm{Rh})$, aluminium oxide $\left(\mathrm{Al}_{2} \mathrm{O}_{3}\right)$, and copper $(\mathrm{Cu})$.

\section{Materials and methods}

The DDA method is used for calculating far-field electromagnetic radiation scattering, absorption, and extinction for targets of any arbitrary shapes, having complex refractive indices and sizes on the order or smaller than incident wavelength [24,25]. In the DDA method, the arbitrary-shaped target is divided into a cubic lattice of $N$ dipoles, where $N$ is the number of dipoles. The inter-dipole separation is small enough compared to the target length and the ambient wavelength. Draine and Flatau [25] proved that the DDA method is applicable if $|m| k d<<1$, where $m$ is the complex refractive index, $k$ is the wavenumber at the ambient medium, and $d$ is the above-mentioned inter-dipole separation. To satisfy the condition of $|m| k d<<1$, and considering the refractive index of the materials and wavelength ranges, the minimum value of the diploe-space is $0.04 \mathrm{~nm}$. We use DDSCAT code, version 7.3, and Lattice Dispersion Relation (LDR) [27] for polarizeability in the DDA simulations. The excitation wave is assumed to propagate along the $x$ axis (direction of the graphene thickness) while electric field is parallel to the $y$ axis (normal to the direction of the graphene thickness). Note that for plasmonic nanoparticles, the accuracy issue can be satisfied for the conditions that dipoles are very small compared to the lengths of characteristics. To prove accuracy of the simulation, a spherical gold nanoparticle of $10 \mathrm{~nm}$ diameter is simulated, which has already been studied by Yurkin et al [28]. We use 383336 dipoles to model the gold nanoparticle. The absorption efficiency spectrum for the $10-\mathrm{nm}$ gold particle is calculated. The results are in great agreement with the results presented in [28]. The computational approaches for plasmonics and their accuracy issues can be found in $[28,29]$. In Figure 1, the calculated refractive indices are shown for graphene that are used in both DDA and FDTD methods. Figure 2 illustrates the proposed structures, a monolayer graphene nanosheet (Figure 2(a)), and graphene nanopore (Figure 2(b)). Also, Figure 2(c) shows the nanopore structure with a nanoparticle inserted into the pore. The structures are illuminated with a transverse-electric field, propagating along the $x$ axis, in Figure 2. The experimental results in $[30,31]$ for the refractive indices are utilized for modelling of monolayer graphene between the 150 and $500-n m$ wavelengths in the DDA method [24,32]. Also, we use the frequency-dependent optical properties of metals from $[33,34]$. The accuracy issues for the thin

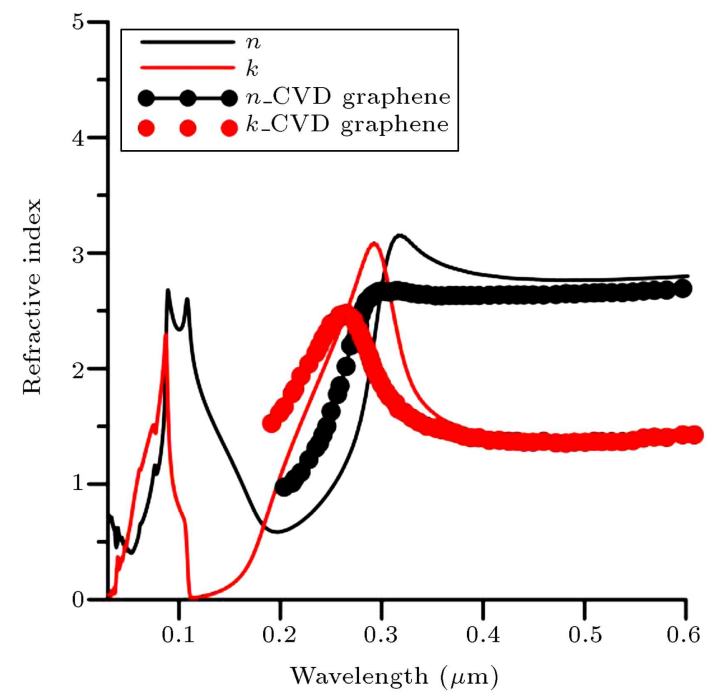

Figure 1. The real and imaginary parts of refractive indices shown for the calculated and experimental CVD graphenes.

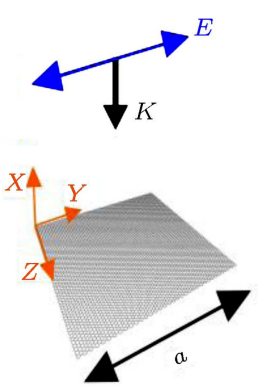

(a)
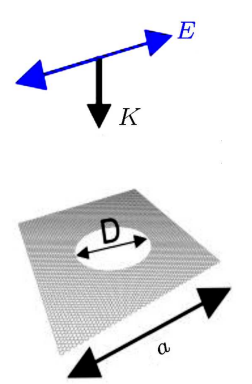

(b)
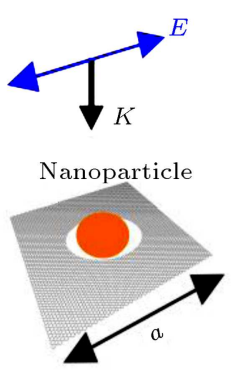

(c)
Figure 2. The proposed structures: (a) Graphene nanosheet, length $a$, (b) graphene nanopore, length $a$, pore diameter $D$, and (c) a nanoparticle is inserted into the graphene nanopore. A plane wave is illuminated in each structure while electric field component $E$ is parallel to the $y$ axis and it propagates along the $x$ axis $(k \| x)$. 


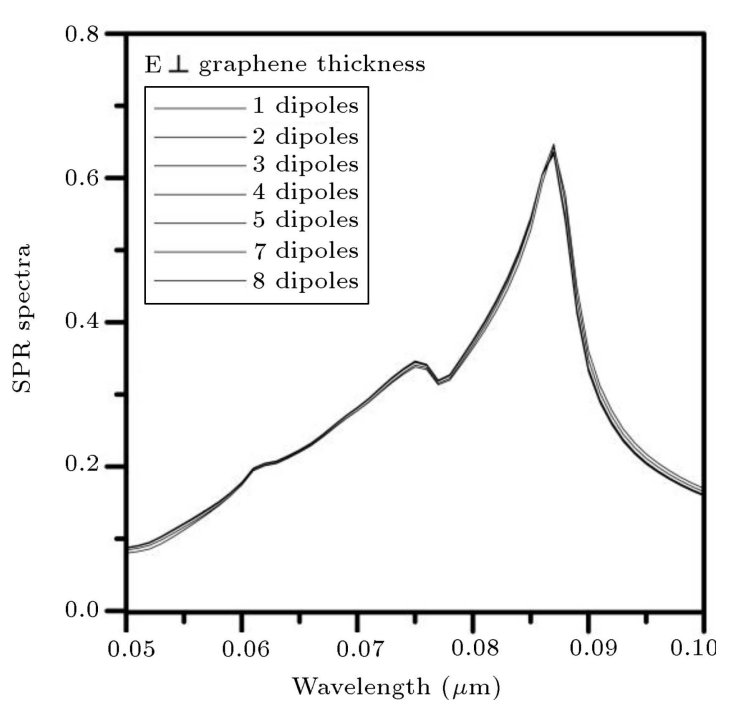

Figure 3. Effect of number of dipoles in the direction of the graphene thickness on the SPR spectra. Results show that increasing number of dipoles from 1 to 8 has almost no effect on the SPR peaks.

layers are important when transverse polarization state is used (electric field is parallel to the direction of the graphene thickness). But, we only consider the polarization state in which the electric field component is normal to the direction of the graphene thickness. In this case, the electric field is almost insensitive to the thickness of the graphene for very thin sheets. The simulation setup is designed to calculate the SPR spectrum for a graphene sheet of $3 \mathrm{~nm}$ length while the number of dipoles in the direction of graphene thickness is changed between 1 and 8. As shown in Figure 3, the number of dipoles has almost no effects on the SPR spectra. For further studies, the thickness-accuracy issues can be found in $[35,36]$.

We need optical properties of graphene for the wavelengths below $100 \mathrm{~nm}$. Thus, we calculate the refractive index of monolayer graphene by using DFT framework implemented in GPAW code [37]. The calculation is carried out considering the Local-Density Approximation (LDA) for exchange-correlation potential plus the linear response of the Random-Phase Approximation (RPA) [38,39]. The unit cell is the primitive cell of graphene containing two carbon atoms with experimental parameter of $a \approx 4.651$ (atomic unit). We consider vertical distance between replicas of graphene to be $L=5 a \approx 23.255$ (atomic unit) to avoid interactions among them. We use $200 \mathrm{eV}$ energy cut-off for plane-wave representation and employ $16 \times 16 \times 1$ and $64 \times 64 \times 1 \mathrm{k}$-point meshes in the MonkhorstPack scheme for ground state and linear response function calculations, respectively. The structure of freestanding graphene is fully relaxed until the maximum force applied to each atom is less than $0.05 \mathrm{eV} / \AA$. Optical broadening is assumed to be $0.2 \mathrm{eV}$ and the response function is evaluated in the optical limit within RPA. Our obtained values for graphene are close to the experimental results in [32] for the ultraviolet and blue-light frequencies (150 $\mathrm{nm}$ to $500 \mathrm{~nm}$ ). To compare the calculated refractive indices with the experimental results, Figure 1 compares calculated and experimental refractive indices for graphene. It can be discussed in two regimes: below $200 \mathrm{~nm}$ and above $200 \mathrm{~nm}$ of wavelength. For the wavelength above $200 \mathrm{~nm}$, the calculated and experimental refractive indices are shown, which are in good agreement. Note that the small differences between the calculated and experimental refractive indices are related to the Chemical Vapor Deposition (CVD) method used for graphene deposition in [31]. These differences are generally due to the experimental methods used for graphene sheet production and optical measurements setups. For wavelengths less than $200 \mathrm{~nm}$, there is no experimental data for dielectric function of graphene at petahertz-frequency range and we should validate our results based on longer wavelengths (wavelengths above $200 \mathrm{~nm}$ in Figure 1). Finally, to investigate SPR and field enhancement properties of the proposed structures at the petahertz frequencies, we use the calculated refractive indices in the DFT framework for modelling graphene sheet in the DDSCAT7.3 code developed in [25]. In the FDTD simulations, the graphene structure is modeled as a sheet with $0.34 \mathrm{~nm}$ of thickness. Refractive indices for the graphene are calculated here and compared with the values in $[26,31,33,34]$. A plane wave is illuminated for the graphene sheet, which propagates along the graphene thickness direction, and electric field component is normal to the direction of graphene thickness. The real-space grid for FDTD is about $0.005 \mathrm{~nm}$ in all directions. The FDTD results are in good agreement with the results of electric-field distribution and field enhancements previously reported in $[11,23]$. The timestep is $0.1 \mathrm{fs}$ and simulations are done for $1000 \mathrm{fs}$. The number of 12 Perfectly Matched Layers (PML) in all directions are used for the boundaries. The boundaries are far enough $(1 \mu \mathrm{m})$ for the object of interest in all directions.

\section{Results and discussion}

\subsection{SPR and EFE properties of graphene nanosheet}

The SPR spectra of the graphene nanosheet with a fixed thickness $(0.34 \mathrm{~nm})$, for different length values (a) from 1 to $15 \mathrm{~nm}$, are shown in Figure 4 . We obtain longitudinal and transverse modes by setting the direction of the $x$ axis and the polarization direction (direction of the electric field) to zero or $90^{\circ}$, respectively (Figure 2). Our simulation results are provided for the transverse mode, because in monolayer graphene, the transverse mode dominates and the 


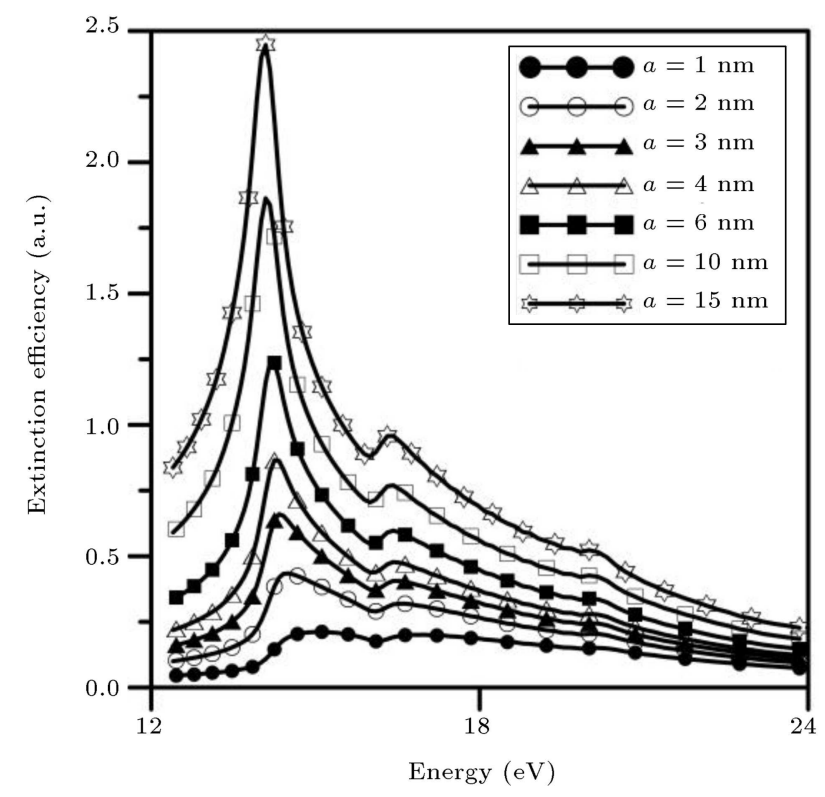

Figure 4. SPR spectra of the graphene nanosheet as a function of the sheet length. There is only one dominant mode $\left(M_{1}\right)$, which is red-shifted by increasing the sheet length.

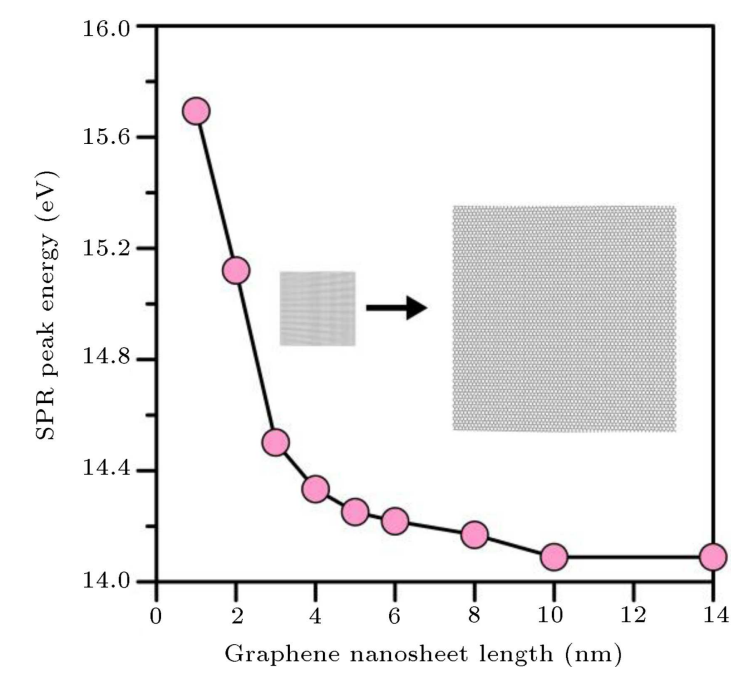

Figure 5. Energy of the SPR peak for the graphene nanosheet as a function of the sheet length. For small sheets, energy decreases and for larger sheets, it is unchanged. Energy of the SPR peak drops from 15.1 to $14.09 \mathrm{eV}$ by increasing the length from 1 to $15 \mathrm{~nm}$.

longitudinal mode can be neglected [11,12,24]. As shown in Figure 4, the SPR spectra have one dominant mode $\left(M_{1}\right)$ and corresponding energy decreases from 15.7 to $14.1 \mathrm{eV}$ when the nanosheet length increases from 1 to $14 \mathrm{~nm}$. The energy of the SPR peak $\left(E_{\max }\right)$ of the transverse-mode SPR as a function of length of the monolayer graphene nanosheet is shown in Figure 5. When the nanosheet length is more than $6 \mathrm{~nm}$, energy of the SPR peak approaches a saturation value and for the length values above $10 \mathrm{~nm}$, it is nearly constant $(14.1 \mathrm{eV})$. This plasmon peak occurs at the petahertz frequency range, which corresponds to the interband $\pi+\sigma$ plasmon of graphene [14-16]. The high-energy interband $\pi+\sigma$ plasmons can be excited in pristine and doped graphene [37]. The SPR spectra of the graphene nanosheet have a peak at the petahertz frequency, which is a combination of the collective and single excitations of all valence electrons [14]. Plasmon peak mainly depends on the size and shape of the target [20,24,40-44]. The energy of the plasmon peak of the monolayer graphene nanodisc, and spherical and cylindrical metals decreases with increasing diameter $[24,43]$. The saturating behaviour of the interband $\pi+\sigma$ plasmon by increasing the sheet length is understandable due to great plasmon damping occurring as a result of equal values of the energy of the interband $\pi+\sigma$ plasmon and electron transitions. This behaviour is observed for the graphene sheet length values above $6 \mathrm{~nm}$, as shown in Figure 5. The interband plasmons cannot move across a long distance in graphene; therefore, they are strongly localized, sensitive to small and insensitive to large sizes [23]. Graphene nanosheets change the external electromagnetic radiations to the enhanced fields by means of localized interband plasmons. The Electric Field Enhancement Factor (EFEF) is defined as $|E|^{2} /\left|E_{0}\right|^{2}$, where $E$ is the electric field at the destination point and $E_{0}$ is the incident electric field. Figure 6 shows the maximum EFEF at the hot spot of the graphene nanosheet as a function of the sheet length from 1 to $30 \mathrm{~nm}$. The maximum EFEF value is 22.01, which corresponds to $a=3 \mathrm{~nm}$. Also,

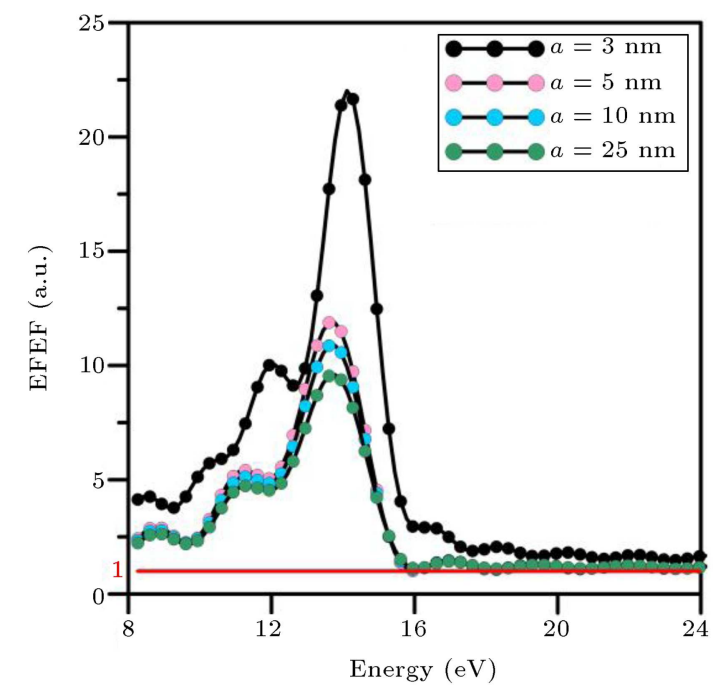

Figure 6. Maximum EFEF as a function of the graphene nanosheet length at the hot spot of the graphene nanosheet. The maximum EFEF value is 22.01, which corresponds to $a=3-\mathrm{nm}$ graphene nanosheet. The inset shows the field profile for $a=1-\mathrm{nm}$ graphene nanosheet at $\lambda=92 \mathrm{~nm}(13.48 \mathrm{eV})$ and the maximum EFEF is 10.7. 


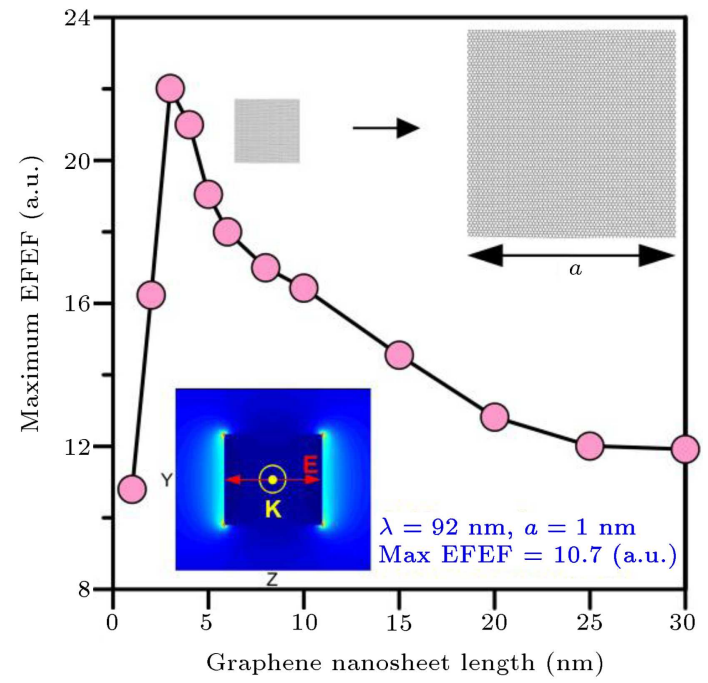

Figure 7. EFEF at the hot spot of the graphene nanosheet as a function of the energy of the incident light for some nanosheet length values $(a=3,5,10$, and 25 $\mathrm{nm}$ ). Longer sheet lengths lead to smaller maximum values of the EFEF.

Figure 7 presents the EFEF at the hot spot of the graphene nanosheet as a function of the energy of incident light for some nanosheet length values between 1 and $15 \mathrm{~nm}$.

\subsection{SPR and EFE properties of graphene nanopore}

Figure 8 represents energy of the SPR peak in the SPR spectra of the graphene nanopore. Making a pore into the graphene nanosheet introduces excess modes to the SPR spectra. When pore diameter increases, energy of the first and second modes is raised due to

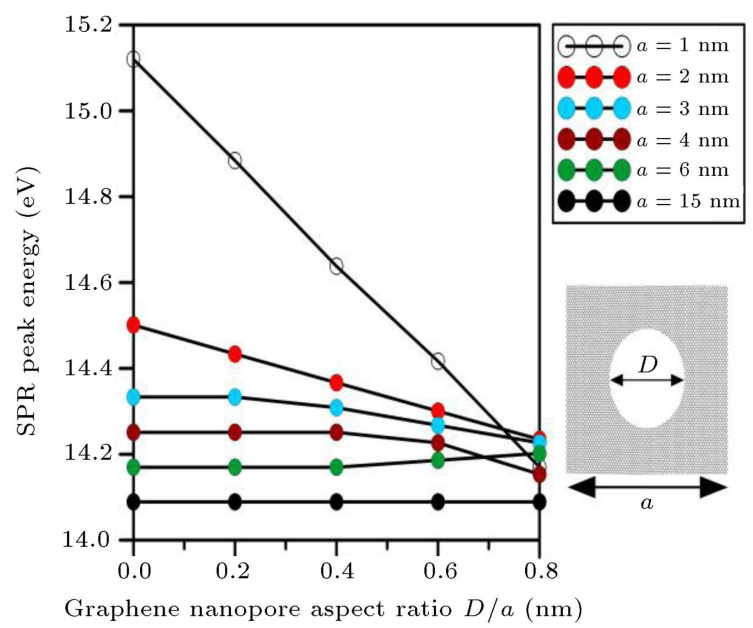

Figure 8. Effect of the pore diameter variations on the energy of the SPR for different graphene sheet sizes. When the pore diameter increases, for small enough sheets, energy of the global peak decreases. For larger sheets, changing the pore diameter has no effect and global peak is unchanged. the decrease cluster size and, consequently, increase in the bandgap energy. But, for the energy value of the third mode, increasing the pore diameter drops corresponding energy. As a result, for small enough sheets $(a<5 \mathrm{~nm})$, the shift amount of energy of the third mode dominates and energy of the global SPR peak decreases (Figure 8). However, for some cases, i.e. $a>5 \mathrm{~nm}$ and $a<8 \mathrm{~nm}$, increasing the pore diameter slightly increases energy of the SPR peak (e.g., $a=6 \mathrm{~nm}$, Figure 8). For larger sheets, small pore diameters have no effect on the energy of the first and second modes. For bigger pore diameters in the big sheets, energy of the global peak is unchanged (Figure 8). However, if $D \approx a$, then energy of the global peak increases. For example, if $D=2.5 \mathrm{~nm}$ while $a=3 \mathrm{~nm}$, then energy of the global peak is about $14.42 \mathrm{eV}$. The EFEF of the graphene nanopore is considered under an external transverse-electric field propagating in the direction of the graphene thickness. Figure 9 presents the EFEF for the hot spot of the 3-nm graphene sheet as a function of energy for the pore diameter changes between 1 and $2 \mathrm{~nm}$. Generally, when the pore diameter increases, the peak value of the EFEF decreases and it is broadened.

Table 1 provides a brief description of the interband $\pi$ and $\pi+\sigma$ plasmon properties in graphene nanosheet and nanopore. The $\pi$ plasmon belongs to the UV-visible (150 to $400 \mathrm{~nm}$ ) region of light while interband $\pi+\sigma$ plasmon appears at the petahertz frequencies $(\sim 80 \mathrm{~nm})$. The interband $\pi$ plasmon properties of graphene nanodiscs and nanosheets are investigated by the DDA method $[11,12,25]$. The saturation energy of the $\pi+\sigma$ plasmon is $14.09 \mathrm{eV}$ and for $\pi$ plasmon, it is $4.35 \mathrm{eV}$. Energy of the SPR peak decreases by increasing the sheet length or disk

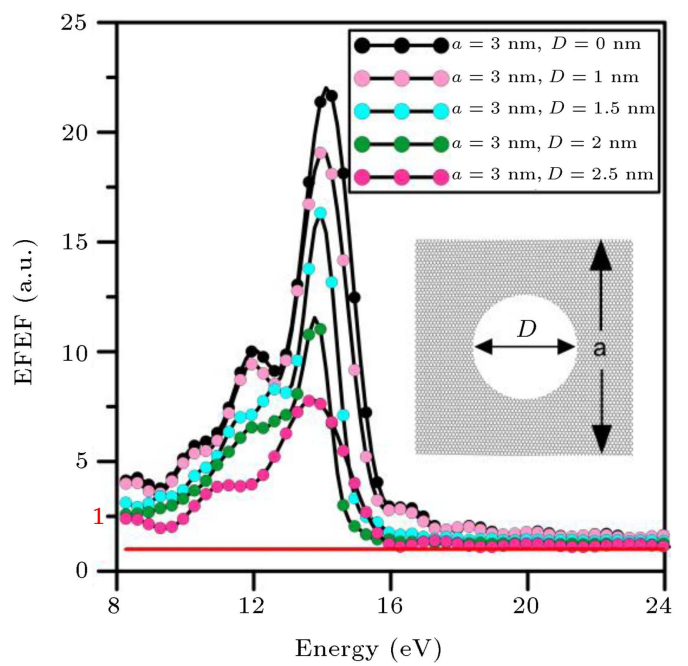

Figure 9. EFEF of the hot spot for a 3-nm graphene sheet as a function of the energy of the incident light and pore diameter. Increasing the pore diameter decreases the maximum value of the EFEF. 
Table 1. Small-size effects and electric-field enhancement properties; comparison between interband $\pi$ and $\pi+\sigma$ plasmons. Dependency of the energy of $\pi$ and $\pi+\sigma$ plasmon peaks and their EFEF properties on the graphene nanosheet length and graphene nanopore diameter.

\begin{tabular}{lcc}
\hline & $\boldsymbol{\pi}$ plasmon $[\mathbf{1 1 , 2 3}]$ & $\boldsymbol{\pi}+\boldsymbol{\sigma}$ plasmon \\
\hline Saturation Energy $(\mathbf{e V})$ & 4.35 & 14.09 \\
Sheet length corresponding to the saturation energy $(\mathbf{n m})$ & 80 & 8 \\
Energy of SPR peak for $3-\mathbf{n m}$ sheet $(\mathbf{e V})$ & 4.96 & 14.5 \\
The largest pore-sensitive sheet $(\mathbf{n m})$ & 60 & 6 \\
Maximum EFEF & 19 & 22 \\
Sheet length corresponding to the maximum EFEF $\mathbf{( n m )}$ & 5 & 3 \\
\hline
\end{tabular}

diameter $[11,12,25]$. The above-mentioned saturation energy for $\pi+\sigma$ and $\pi$ plasmons belongs to $a=8$ and 80 -nm graphene sheets, respectively. For a graphene nanosheet length of $a=3 \mathrm{~nm}$, the energy of $\pi+\sigma$ and $\pi$ plasmons is 4.96 and $14.5 \mathrm{eV}$, respectively. Making a pore into the graphene nanosheet reduces energy of the SPR peak. For large enough sheets, the pore diameter variations cannot change the energy of the SPR peak, except for the case in which the pore diameter is almost equal to the nanosheet length. The largest poresensitive sheet length is defined as the largest graphene nanosheet length that makes a small pore within it $\left(\frac{D}{a} \leq 0.8\right)$, resulting in a considerable shift in the SPR spectra. This factor is 60 and $6 \mathrm{~nm}$ for interband $\pi$ and $\pi+\sigma$ plasmons, respectively. The maximum EFEF for interband $\pi$-plasmon is 8.5 for monolayer graphene nanodisc, which belongs to $8 \mathrm{~nm}$-diameter disk [25]. Also, for graphene nanosheet, this value is 19.8 and belongs to $a=5 \mathrm{~nm}[11,12]$. The maximum EFEF for interband $\pi+\sigma$ plasmon is 22 for graphene nanosheet, which belongs to $a=3 \mathrm{~nm}$. Generally, our results here prove that the general behaviours of the two different types of interband plasmons in graphene nanosheet and nanopore are similar, except for the absolute values of the energy and EFEF.

Both plasmons can be used in single-molecule or nanoscale-objects detections. For example, interband $\pi$ plasmon has been employed for DNA nucleotide sensing; the method can clearly distinguish all the possible types of the DNA nucleotides. Here, we examined $\pi+\sigma$ and we proved that this kind of plasmon was not applicable in DNA sequencing and it could not distinguish types of the presented DNA nucleotides. However, this is not a drawback for this kind of plasmon, because this happens due to the similar optical properties of the DNA nucleotides in the petahertz frequency range. Thus, the interband $\pi+\sigma$ plasmon in graphene nanopore may be used in other singlemolecule detection mechanisms. Hence, application of interband $\pi+\sigma$ SPR in graphene nanopore for detecting nanoparticles is examined. Here, we study the applicability of the proposed petahertz-frequency plasmons to be used in nanoscale-objects detections. The idea

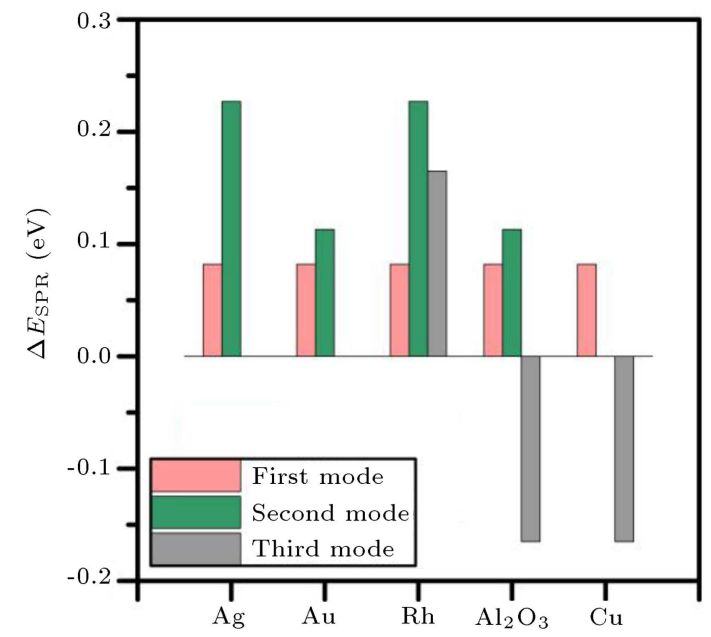

Figure 10. Effects of different types of the nanoparticle presented to the graphene nanpore, which introduce energy shifts to the modes of the plasmon spectra. As presented here, the $\mathrm{Ag}, \mathrm{Au}, \mathrm{Rh}, \mathrm{Cu}$, and $\mathrm{Al}_{2} \mathrm{O}_{3}$ nanoparticles can be distinguished by looking at the energy shifts in these spectra.

is based on the fact that interband $\pi+\sigma$ plasmon in graphene is strongly localized and sensitive to small dimensions of the target. We examine applicability of the interband plasmons by presenting $2 \mathrm{~nm}$-diameter particles of gold $(\mathrm{Au})$, silver $(\mathrm{Ag})$, rhodium $(\mathrm{Rh})$, copper $(\mathrm{Cu})$, and aluminium oxide $\left(\mathrm{Al}_{2} \mathrm{O}_{3}\right)$ to a $3 \mathrm{~nm}$ length graphene sheet. Figure 10 represents the energy shifts in the SPR spectra related to each SPR mode due to the presented nanoparticles. As shown in Figure 10, the suggested mechanism is selective between types of the presented nanoparticle. Hence, type of the presented nanoparticle can be clearly determined only by utilizing energy shifts of the petahertz-frequency SPR spectra. For example, if the shift value for the second mode of the SPR spectra is zero, then the presented nanoparticle is $\mathrm{Cu}$. If the shift value for the third mode is zero, then we look at the shift value for the second mode. In this condition, if the shift value of the energy of the second mode is about $0.2 \mathrm{eV}$, the presented nanoparticle is $\mathrm{Ag}$ and if the shift value is about $0.1 \mathrm{eV}$, the presented nanoparticle is $\mathrm{Au}$. If we 
see positive and non-zero energy shifts in all the three modes, the presented nanoparticle is Rh. Finally, if the first and second mode energies are shifted positively while the third mode energy is shifted negatively, the presented nanoparticle is $\mathrm{Al}_{2} \mathrm{O}_{3}$. As demonstrated here, interband $\pi+\sigma$ plasmon in graphene nanopore can be used in determining types of the presented nanoparticle to the pore. Here, we examined five different types of the nanoparticles, which were clearly distinguished by the proposed method. The proposed method can be used in the nanoparticle devices as a new methodology for nanoscale-object sensing, counting, or manipulating.

\section{Conclusions}

In summary, we have explored the SPR and fieldenhancement properties of the interband $\pi+\sigma$ plasmon (petahertz-frequency plasmon) in monolayer graphene nanosheet and graphene nanopore. First, we have calculated the refractive indices for graphene at the petahertz frequency range by employing DFT framework. Using DDA method, we have shown that for graphene nanosheets smaller than $6 \mathrm{~nm}$, energy of the SPR peak decreases with increasing length of the sheet and for values above $10 \mathrm{~nm}$, it is unchanged. Then, we have looked into the effect of making a nanopore at the center of the sheet. By increasing diameter of the pore, energy of the SPR peak drops. For the large graphene sheets (bigger than $8 \mathrm{~nm}$ ), variations of the pore diameter have no effect on the SPR spectra. The interband $\pi+\sigma$ plasmon is strongly localized and small-size-sensitive. Hence, it can be used as a promising method for a new nanoscale-object detection methodology. We have examined this applicability by nanoscale particles of $\mathrm{Ag}, \mathrm{Au}, \mathrm{Rh}, \mathrm{Cu}$, and $\mathrm{Al}_{2} \mathrm{O}_{3}$. They introduce 0.195 to $0.417 \mathrm{eV}$ shifts in the SPR spectra. As a result of our study, type of the presented nanoparticle can be clearly determined and the method has proved that it can be used in nanoscale-objects sensing, counting, or manipulating.

\section{Acknowledgements}

Bashir Fotouhi thanks Omar Moradi for sharing valuable information on the DDA method. The authors also acknowledge the Iran Nanotechnology Initiative Council (INIC) for the partial support of this project.

\section{Abbreviations}

$\begin{array}{ll}\mathrm{Ag} & \text { Silver } \\ \mathrm{Al}_{2} \mathrm{O}_{3} & \text { Aluminium Oxide } \\ \mathrm{Au} & \text { Gold } \\ \mathrm{Cu} & \text { Copper }\end{array}$

$\begin{array}{ll}\text { DDA } & \text { Discrete Dipole Approximation } \\ \text { DFT } & \text { Density Functional Theory } \\ \text { DNA } & \text { Deoxyribonucleic acid } \\ \text { EELS } & \text { Electron Energy Loss Spectroscopy } \\ \text { EFEF } & \text { Electric Field Enhancement Factor } \\ \text { eV } & \text { Electron-Volt } \\ \text { FDTD } & \text { Finite Difference Time Domain } \\ \text { Hz } & \text { Hertz } \\ \text { LDA } & \text { Local Density Approximation } \\ \text { nm } & \text { Nanometer } \\ \text { Rh } & \text { Rhodium } \\ \text { RPA } & \text { Random Phase Approximation } \\ \text { SPR } & \text { Surface Plasmon Resonance } \\ \text { UV } & \text { Ultraviolet }\end{array}$

\section{References}

1. He, S., Song, B., Li, D., Zhu, C., Qi, W., Wen, Y., Wang, L., Song, S., Fang, H. and Fan, C. "A graphene nanoprobe for rapid, sensitive, and multicolor fluorescent DNA analysis", Adv. Funct. Mater., 20(3), pp. 453-459 (2010).

2. Luo, X., Qiu, T., Lu, W. and Ni, Z. "Plasmons in graphene: recent progress and applications", Mater. Sci. Eng., R., 74(11), pp. 351-376 (2013).

3. Stauber, T. "Plasmonics in Dirac systems: from graphene to topological insulators", J. Phys.: Condens. Matter., 26(12), p. 123201 (2014).

4. Wang, X., Meng, G., Zhu, C., Huang, Z., Qian, Y., Sun, K. and Zhu, X. "A generic synthetic approach to large-scale pristine-graphene/metal-nanoparticles hybrids", Adv. Funct. Mater., 23(46), pp. 5771-5777 (2013).

5. Huang, Q., Kim, J.J., Ali, G. and Cho, S.O. "Widthtunable graphene nanoribbons on a $\mathrm{SiC}$ substrate with a controlled step height", Adv. Mater., 25(8), pp. 11441148 (2013).

6. Geng, X., Niu, L., Xing, Z., Song, R., Liu, G., Sun, M., Cheng, G., Zhong, H., Liu, Z., Zhang, Z. and Sun, L. "Aqueous-processable noncovalent chemically converted graphene-quantum dot composites for flexible and transparent optoelectronic films", Adv. Mater., 22(5), pp. 638-642 (2010).

7. Garaj, S., Hubbard, W., Reina, A., Kong, J., Branton, D. and Golovchenko, J. A. "Graphene as a subnanometre trans-electrode membrane", Nature, 467(7312), pp. 190-193 (2010).

8. Habibi, M., Darbari, S., Rajabali, S. and Ahmadi, V. "Fabrication of a graphene-based pressure sensor by utilising field emission behavior of carbon nanotubes", Carbon, 96, pp. 259-267 (2016).

9. Koohi, M.Z. and Neshat, M. "Evaluation of graphenebased terahertz photoconductive antennas", Sci. Iran. Trans. F, 22(3), p. 1299 (2015). 
10. Schneider, G.F., Kowalczyk, S.W., Calado, V.E., Pandraud, G., Zandbergen, H. W., Vandersypen, L.M. and Dekker, C. "DNA translocation through graphene nanopores", Nano Lett., 10(8), pp. 3163-7 (2010).

11. Fotouhi, B., Ahmadi, V. and Faramarzi, V. "Nanoplasmonic-based structures for DNA sequencing", Opt. Lett., 41(18), pp. 4229-4932 (2016).

12. Fotouhi, B., Ahmadi, V., Abasifard, M. and Roohi, R. "Interband $\pi$ plasmon resonance of graphene nanopores: a potential sensing mechanism for DNA sensing", J. Phys. Chem. C, 120(25), pp. 13693-13700 (2016).

13. Hu, J., Wang, L., Cai, W., Li, Y., Zeng, H., Zhao, L. and Liu, P. "Smart and reversible surface plasmon resonance responses to various atmospheres for silver nanoparticles loaded in mesoporous $\mathrm{SiO}_{2}$ ", J. Phys. Chem. C., 113(44), pp. 19039-19045 (2009).

14. Despoja, V., Novko, D., Dekanić, K., Šunjić, M. and Marušić, L. "Two-dimensional and $\pi$ plasmon spectra in pristine and doped graphene", Phys. Rev. B., 87(7), p. 075447 (2013).

15. Kinyanjui, M.K., Kramberger, C., Pichler, T., Meyer, J.C., Wachsmuth, P., Benner, G. and Kaiser, U. "Direct probe of linearly dispersing 2D interband plasmons in a free-standing graphene monolayer", EPL., $\mathbf{9 7}(5)$, p. 57005 (2012).

16. Politano, A., Marino, A.R., Formoso, V., Farías, D., Miranda, R. and Chiarello, G. "Quadratic dispersion and damping processes of $\pi$ plasmon in monolayer graphene on Pt (111)", Plasmonics, 7(2), pp. 369-376 (2012).

17. Generalov, A.V. and Dedkov, Y.S. "EELS study of the epitaxial graphene/Ni (111) and graphene/Au/Ni (111) systems", Carbon, 50(1), pp. 183-191 (2012).

18. Marinopoulos, A.G., Reining, L., Rubio, A. and Olevano, V. "Abinitio study of the optical absorption and wave-vector-dependent dielectric response of graphite", Phys. Rev. B., 69(24), p. 245419 (2004).

19. Novko, D., Despoja, V. and Šunjić, M. "Changing character of electronic transitions in graphene: from single-particle excitations to plasmons", Phys. Rev. B., 91(19), p. 195407 (2015).

20. Thongrattanasiri, S., Manjavacas, A. and García de Abajo, F.J. "Quantum finite-size effects in graphene plasmons", ACS Nano, 6(2), pp. 1766-1775 (2012).

21. Alonso-González, P., Nikitin, A.Y., Golmar, F., Centeno, A., Pesquera, A., Vélez, S., Chen, J., Navickaite, G., Koppens, F., Zurutuza, A. and Casanova, F. "Controlling graphene plasmons with resonant metal antennas and spatial conductivity patterns", Science, 344(6190), pp. 1369-1373 (2014).

22. Cocchi, C., Prezzi, D., Ruini, A., Benassi, E., Caldas, M.J., Corni, S. and Molinari, E. "Optical excitations and field enhancement in short graphene nanoribbons", J. Phys. Chem. Lett., 3(7), pp. 924-929 (2012).

23. Zhou, W., Lee, J., Nanda, J., Pantelides, S.T., Pennycook, S.J. and Idrobo, J.C. "Atomically localized plasmon enhancement in monolayer graphene", Nat. Nanotechnol., 7(3), pp. 161-165 (2012).

24. Hu, J., Zeng, H., Wang, C., Li, Z., Kan, C. and Liu, Y. "Interband $\pi$ plasmon of graphene: strong small-size and field-enhancement effects", Phys. Chem. Chem. Phys., 16(42), pp. 23483-23491 (2014).

25. Draine, B.T. and Flatau, P.J. "User guide for the discrete dipole approximation code DDSCAT 7.0", 2008, available at https://arxiv.org/abs/0809.0337.

26. Yee, K.S. "Numerical solution of initial boundary value problems involving Maxwell's equations in isotropic media", IEEE Trans. Antennas Propag., 14(3), pp. 302-307 (1966).

27. Yurkin, M.A. and Hoekstra, A.G. "The discrete dipole approximation: an overview and recent developments", J. Quant. Spectrosc. Radiat. Transfer, 106(1), pp. 558-589 (2007).

28. Yurkin, M.A., de Kanter, D. and Hoekstra, A.G. "Accuracy of the discrete dipole approximation for simulation of optical properties of gold nanoparticles", J. Nanophoton, 4(1), p. 41585 (2010).

29. Yurkin, M.A. "Computational approaches for plasmonics", Handbook of Molecular Plasmonics, pp. 83135 (2013).

30. Kravets, V.G., Grigorenko, A.N., Nair, R.R., Blake, P., Anissimova, S., Novoselov, K.S. and Geim, A.K. "Spectroscopic ellipsometry of graphene and an exciton-shifted van Hove peak in absorption", Phys. Rev. B., 81(15), p. 155413 (2010).

31. Nelson, F.J., Kamineni, V.K., Zhang, T., Comfort, E.S., Lee, J.U. and Diebold, A.C. "Optical properties of large-area polycrystalline chemical vapor deposited graphene by spectroscopic ellipsometry", Appl. Phys. Lett., $97(25)$, p. 253110 (2010).

32. Amendola, V. "Surface plasmon resonance of silver and gold nanoparticles in the proximity of graphene studied using the discrete dipole approximation method", Phys. Chem. Chem. Phys., 18(3), pp. 2230-2241 (2016).

33. Johnson, P.B. and Christy, R.W. "Optical constant of transition metals: Ti, V, Cr, Mn, Fe, Co, Ni, and Pd", Phys. Rev., 9(12), pp. 5056-70 (1974).

34. Alcaraz, O.R., Sanz, J.M., Barreda, A.I., Saiz, J.M., González, F., Everitt, H.O. and Moreno, F. "Rhodium tripod stars for UV plasmonics", J. Phys. Chem. C., 119(22), pp. 12572-80 (2015).

35. Wojtkiewicz, S., Sawosz, P., Kostecki, M. and Sokolowska, A. "Optical method for characterization of nanoplates in lyosol", Microel. Engin., 108, pp. 121126 (2013).

36. Smunev, D.A., Chaumet, P.C. and Yurkin, M.A."Rectangular dipoles in the discrete dipole 
approximation", J. Quant. Spectrosc. Radiat. Transfer, 156, pp. 67-79 (2015).

37. Enkovaara, J., Rostgaard, C., Mortensen, J.J., Chen, J., Dulak, M., Ferrighi, L., Gavnholt, J., Glinsvad, C., Haikola, V., Hansen, H.A. and Kristoffersen, H.H. "Electronic structure calculations with GPAW: a realspace implementation of the projector augmentedwave method", Journal of Physics: Condensed Matter, 22(25), p. 253202 (2010).

38. Perdew, J.P. and Zunger, A. "Self-interaction correction to density-functional approximations for manyelectron systems", Phys. Rev. B., 23(10), p. 5048 (1981).

39. Yan, J., Mortensen, J.J., Jacobsen, K.W. and Thygesen, K.S. "Linear density response function in the projector augmented wave method: applications to solids, surfaces, and interfaces", Phys. Rev. B., 83(24), p. 245122 (2011).

40. Hwang, S.W., Shin, D.H., Kim, C.O., Hong, S.H., Kim, M.C., Kim, J., Lim, K.Y., Kim, S., Choi, S.H., Ahn, K.J. and Kim, G. "Plasmon-enhanced ultraviolet photoluminescence from hybrid structures of graphene/ZnO films", Phys. Rev. Lett., 105(12), p. 127403 (2010).

41. Ghorbanzadeh, M., Darbari, S. and Moravvej-Farshi, M.K. "Graphene-based plasmonic force switch", App. Phys. Lett., 108(11), p. 111105 (2016).

42. Amendola, V., Bakr, O.M. and Stellacci, F. "A study of the surface plasmon resonance of silver nanoparticles by the discrete dipole approximation method: effect of shape, size, structure, and assembly", Plasmonics, 5(1), pp. 85-97 (2010).

43. Hu, J., Chen, L., Lian, Z., Cao, M., Li, H., Sun, W., Tong, N. and Zeng, H. "Deep-ultraviolet-blue-light surface plasmon resonance of $\mathrm{Al}$ and $\mathrm{Al}$ core $/ \mathrm{Al}_{2} \mathrm{O}_{3}$ Shell in spherical and cylindrical nanostructures", $J$. Phys. Chem. C., 116(29), pp. 15584-15590 (2012).

44. Politano, A. and Chiarello, G. "Plasmon modes in graphene: status and prospect", Nanoscale, 6, pp. 10927-10940 (2014).

\section{Biographies}

Bashir Fotouhi was born in Sanandaj, Iran, in 1988. He received MSc degree in Electrical Engineering from Tarbiat Modares University (TMU) in 2012 and has been working towards his $\mathrm{PhD}$ degree at TMU since
2012. His professional interests focus on optoelectronics, bio-photonics, graphene plasmonics, nanopore DNA sequencing, and pattern recognition; his current projects include graphene plasmonics and nanopore DNA sequencing.

Vahid Ahmadi (M'86, SM'09) received the PhD degree in Electronic Engineering from the Kyoto University, Japan, in 1994. He is a Professor of Electronic Engineering at Tarbiat Modares University (TMU), Tehran, Iran. He was the Head of the Semiconductor Department of Laser Research Centre, Tehran, from 1994 to 2006. He was the Head of the Electrical Engineering Department of TMU during 2006 to 2008. He was the technical and scientific Chair of the 13th Iranian Conf. on Optics and Photonics, 2007, and the general Chair of the 16th Iranian Conference on Electrical Engineering (ICEE2008), 2008. He is the member of the Founders Board of Optics and Photonics Society of Iran and the editorial board of International Journal of Information \& Communication Technology Research (IJICTR). He is also the Editor-in-Chief of Modares Journal of Electrical Engineering. His current research interests include quantum photonics devices, CNT and GNR based photonic devices, optical modulator and amplifiers, optical microresonator active and passive devices, all optical switches, slow light, photonic crystals, plasmonics, and metamaterial devices.

Mostafa Abasifard was born in 1988, Iran. He received the BS degree from Shahid Beheshti University in 2011 and the MS degree from Tarbiat Modares University, Tehran, Iran, in 2015, both in Electrical Engineering. His research interests include optical and plasmonic properties of graphene nano-structures, nanopore based DNA sequencing, and Ab initio study of low-dimensional systems.

Vahid Faramarzi received the BSc degree from Razi University, Kermanshah, Iran, in 2010, and MSc degree from the Department of Electrical Engineering at Sharif University of Technology, Tehran, Iran, in 2012, both in Electrical Engineering. He is currently working towards the PhD degree in the same field at Tarbiat Modares University, Tehran, Iran. His current research interests include bio-photonics, optoelectronic devices, and graphene based sensors. 